\title{
MERTENS' THEOREM AND SEQUENCE TRANSFORMATIONS
}

\author{
HUGH J. HAMILTON
}

The purposes of this note are to prove by sequence transformation theory a known [ 1 , Theorem 6$]^{1}$ form of Mertens' theorem which admits of a valid converse, and by extending this method to supplement some recent results of Sheffer [2] on extensions of Mertens' theorem to higher dimensions.

Let $\sum_{i=0}^{\infty} a_{i}$ and $\sum_{i=0}^{\infty} b_{i}$ be two convergent series, with sums $A$ and $B$, respectively. We shall refer to these series as " $a$-series" and " $b$-series," respectively. By definition, their "Cauchy-product series" is $\sum_{k=0}^{\infty} \sum_{i=0}^{k} a_{i} b_{k-i}$. We shall refer to this series as the " $c$-series" for the $a$ - and $b$-series. Now Mertens' theorem states that $a$ sufficient condition that the c-series converge to $A B$ is that either the $a$-or the $b$-series converge absolutely. Examples show that the condition is not necessary. On the other hand, J. D. Hill has rephrased the theorem so that its converse is true. ${ }^{2}$

THEOREM 1 A necessary and sufficient condition that the c-series for an a-series and each b-series converge to $A B$ is that the a-series converge absolutely.

In two dimensions our $a-, b$-, and $c$-series become, respectively, $\sum_{i, j=0}^{\infty} a_{i j}, \sum_{i, j=0}^{\infty} b_{i j}$, and $\sum_{k, l=0}^{\infty} \sum_{i, j=0}^{k, k} a_{i j} b_{k-i, l-j}$. We shall again suppose that the first two converge, ${ }^{3}$ and shall denote their sums by $A$ and $B$, respectively. Sheffer has recently shown [2, Theorem 1] that $a$ sufficient condition that the c-series converge to $A B$ is that either the $a$-or the $b$-series converge absolutely and the other series converge boundedly. ${ }^{4}$ In our Theorem 3 (below) we show that a sufficient condition that the $c$-series converge to $A B$ is that either the $a$-or the b-series have only a finite number of nonzero terms. Sheffer's results and ours may be regarded as generalizations of Mertens' theorem, his being the more interesting and ours at first sight trivial.

More interesting than either generalization is the problem of rephrasing it so as to obtain a valid converse. Sheffer in fact does this

Presented to the Society, April 26, 1947; received by the editors February 25, 1947.

${ }^{1}$ Numbers in brackets refer to the papers cited at the end of this article.

${ }^{2}$ We defer proofs to the end.

${ }^{3}$ Convergence of multiple series shall be in the sense of Pringsheim.

${ }^{4}$ That is, with bounded partial sums. 
[2, Theorems 2 and 3] with his generalization to secure the following result.

THEOREM (Sheffer). A necessary and sufficient condition that the $c$-series for a b-series and each absolutely convergent a-series converge to $A B$ is that the b-series converge boundedly.

Unfortunately, this phrasing extends itself neither to the classical Mertens' theorem nor to our generalization. However, the phrasing of Theorem 1 (above) extends itself readily to both Sheffer's and our generalizations, as follows:

THEOREM 2. A necessary and sufficient condition that the c-series for an a-series and each boundedly convergent $b$-series converge to $A B$ is that the a-series converge absolutely.

The duality between Theorem 2 and Sheffer's theorem is interesting. Our simple proof (below) of Theorem 2 of course establishes Sheffer's generalization of Mertens' theorem [2, Theorem 1].

ThEOREM 3. A necessary and sufficient condition that the c-series for an a-series and each convergent b-series converge to $A B$ is that the $a$-serves have only a finite number of nonzero terms.

We now give proofs, remarking by the way that both Sheffer's and our theorems are equally valid for $n$-tuple series with $n>2$ in general [2, proofs of Theorems 1 and 2]. Our proofs are applications of linear sequence transformation theory. First, letting $C_{m} \equiv \sum_{k=0}^{m} \sum_{i=0}^{k} a_{i} b_{k-i}$ and $B_{k} \equiv \sum_{i=0}^{k} b_{i}$, we have

$$
C_{m}=\sum_{i=0}^{m} a_{i} \sum_{k=i}^{m} b_{k-i}=\sum_{i=0}^{m} a_{i} B_{m-i}=\sum_{i=0}^{m} a_{m-i} B_{i} .
$$

Similarly, ${ }^{5}$ letting $C_{m n} \equiv \sum_{k, l=0}^{m, n} \sum_{i, j=0}^{k, l} a_{i j} b_{k-i, l-j}$ and $B_{k l} \equiv \sum_{i, j=0}^{k, b} b_{i j}$, we have

$$
C_{m n}=\sum_{i, j=0}^{m, n} a_{i j} \sum_{k, l=i, j}^{m, n} b_{k-i, l-j}=\sum_{i, j=0}^{m, n} a_{i j} B_{m-i, n-j}=\sum_{i, j=0}^{m, n} a_{m-i, n-j} B_{i j} .
$$

Thus the partial sums of the $c$-series are linear transformations of the partial sums of the $b$-series, both in one and in two dimensions.

Now the well known Silverman-Toeplitz conditions that a summability method be regular provide the following lemma.

LEMMA 1. A necessary and sufficient condition that $\lim _{m \rightarrow \infty} \sum_{i=0}^{m} \alpha_{m-i} B_{i}$

\footnotetext{
${ }^{5}$ See also $[2$, p. $1038,(10)]$.
} 
$=\lim _{i \rightarrow \infty} B_{i}$ whenever the latter exists is that $\sum_{i=0}^{\infty} \alpha_{i}=1$ with $\sum_{i=0}^{\infty}\left|\alpha_{i}\right|<\infty$.

Similarly, we have $[3$, pp. $59-60, B C \rightarrow C$ with preservation of the limit] the following lemma.

LEMMA 2. A necessary and sufficient condition that

$$
\lim _{m, n \rightarrow \infty} \sum_{i, j=0}^{m, n} \alpha_{m-n, n-j} B_{i j}=\lim _{i, j \rightarrow \infty} B_{i j}
$$

whenever the latter exists boundedly is that $\sum_{i, j=0}^{\infty} \alpha_{i j}=1$ with $\sum_{i, j=0}^{\infty}\left|\alpha_{i j}\right|<\infty$.

Again, we have [3, pp. 59-60, $C \rightarrow C$ with preservation of the limit] the following lemma.

LEMMA 3. A necessary and sufficient condition that

$$
\lim _{m, n \rightarrow \infty} \sum_{i, j=0}^{m, n} \alpha_{m-i, n-j} B_{i j}=\lim _{i, j \rightarrow \infty} B_{i j}
$$

whenever the latter exists is that $\sum_{i, j=0}^{\infty} \alpha_{i j} \neq 1$ with all but a finite number of the $\alpha_{i j}$ equal to zero.

We assume for the moment that $A=0$.

Proof $^{6}$ of Theorem 1. Note that $C_{m} / A=\sum_{i=0}^{m}\left(a_{m-i} / A\right) B_{i}$ and apply Lemma 1.

Proof of Theorem 2. Note that $C_{m n} / A=\sum_{i, j=0}^{m, n}\left(a_{m-i, n-j} / A\right) B_{i j}$ and apply Lemma 2.

Proof of Theorem 3. Note that $C_{m n} / A=\sum_{i, j=0}^{m, n}\left(a_{m-i, n-j} / A\right) B_{i j}$ and apply Lemma 3.

If $A=0$ we replace the lemmas by similar ones referring to $[3, \mathrm{pp}$. 49,29 , and 32] and reach our conclusions directly.

In conclusion we remark that the boundedness condition in Lemma 2 and the condition in Lemma 3 that nearly all of the $\alpha_{i j}$ vanish are necessary that the transforms in the respective lemmas be bounded even for all sufficiently large $m$ and $n$, to say nothing of convergence $[3$, p. 41,3 and 4$] .^{7}$

\section{REFERENCES}

1. J. D. Hill, On the space ( $\gamma$ ) of convergent series, Tôhoku Math. J. vol. 45 (1939) pp. 332-337.

2. I. M. Sheffer, Note on multiply infinite series, Bull. Amer. Math. Soc. vol. 52 (1946) pp. 1036-1041.

3. Hugh J. Hamilton, Transformations of multiple sequences, Duke Math. J. vol. 2 (1936) pp. 29-60.

\section{Pomona College}

${ }^{6}$ See also proof of $[1$, Theorem 6$]$.

${ }^{7}$ Compare [1], last phrase in statement of Theorem 2. 